\title{
Ten years analysis of Tropospheric refractivity variations
}

\author{
Stergios A. Isaakidis and Thomas D. Xenos \\ Department of Electrical and Computer Engineering, Aristotle University of Thessaloniki, Greece
}

\begin{abstract}
The refractivity variations of the troposphere are responsible for various effects on radio wave propagation, such as refraction, bending, radio-station interference, etc. In this work, the refractivity variations of the Hellenic troposphere are studied using data from Helliniko airport of Athens/Greece. The data were analyzed using various interpolation procedures, i.e. in a day-by-day manner for temperature and relative humidity, to transform the data according to a reference height common for the whole dataset and finally for the refractivity $N$ with respect to time, using piece-wise hermite interpolation polynomials for the low and medium altitudes and linear interpolation factors for the high altitudes. Since refractivity varies with time and height, two height independent basic parameters were computed and analyzed: the refractivity at station height $N_{0}$ and scale height $H_{s}$. These parameters can be used to calculate the refractivity profile. Using statistical tools as the moving average, the $\beta_{0}$ parameter and the monthly mean values, together with the corresponding standard deviations, useful results were obtained for the variations of the refractivity with respect to observation hour, height, season, month, day and level.
\end{abstract}

Key words troposphere - refractivity - scale height - $\beta_{0}$ parameter

\section{Introduction}

The troposphere is the lower region of Atmosphere and it extends from the earth's surface to an altitude of about $10 \mathrm{~km}$ at the middle latitudes $(6 \mathrm{~km}$ at the poles and up to $18 \mathrm{~km}$ at the equator). The basic parameters that describe the tropospheric medium are pressure, temperature and relative humidity. Propagation in the troposphere is affected by the variations of the refractive index with time and height. These refractivity variations are responsible for various phenomena in the wave propagation, such as range and elevation angle errors in radar acquisition (Lear,

Mailing address: Dr. Stergios A. Isaakidis, Department of Electrical and Computer Engineering, Aristotle University of Thessaloniki, GR-54 124 Thessaloniki, Greece; email: sisaakid@otenet.gr
1980; Anderson, 1995), refraction and fading of the electromagnetic waves (Flock, 1987), ducting phenomena (Patterson et al., 1994), etc.

The index of refraction is defined as $n=\sqrt{\varepsilon_{r}=c / v}$, where $\varepsilon_{r}$ is the dielectric constant of the troposphere, $c$ is the speed of light and $v$ is the phase velocity of the wave in the medium. Because $n$ is slightly greater than unity near the earth's surface (1.00025-1.00040), in practice we use the scaled index of refraction $N$ which is called refractivity and is defined as (Flock, 1987)

$N=(n-1) \times 10^{6}=\frac{77.6 p}{T}-\frac{5.6 e}{T}+\frac{3.75 \times 10^{5} e}{T^{2}}$

where $p$ is the total pressure in mbar, $e$ is the water vapor pressure and $T$ is the temperature in Kelvin degrees.

If the Relative Humidity (RH \%) and temperature are given, the water vapor pressure $e$ can be computed with the following set of equations (Lear, 1980): 


$$
\begin{gathered}
x=\frac{17.2694(T-273.15)}{T-35.85} \\
e_{s}=6.11 \times \exp (x)(\text { mbars }) \\
e=\frac{\mathrm{RH}}{100} e_{s} .
\end{gathered}
$$

Theoretically speaking, the refractivity with respect to height is expressed by the exponential relation

$$
N=N_{0} \exp \left(-h / H_{s}\right)
$$

where $N_{0}$ is the refractivity at the station's height, $h$ is the height and $H_{s}$ is the scale height. It is obvious that the parameters $N_{0}$ and $H_{\mathrm{s}}$ are independent of height and since their changes will be reflected in $N$ according to eq. (1.5), they constitute a useful tool for the study of the refractivity variations.

The purpose of this work is to study the tropospheric refractivity variations over the Hellenic region. These variations describe the tropospheric conditions over the specific region of interest and can be taken into account as an empirical reference for troposheric wave propagation, scale height estimations, bending of rays for terrestrial or satellite paths and so on.

\section{Data analysis}

For the calculation of the refractivity variations, data from Helliniko airport of Athens/ Greece $\left(37^{\circ} 53^{\prime} \mathrm{N}, 23^{\circ} 43^{\prime} \mathrm{E}\right)$ were used. The data cover the period from 1991 to 1999 and the measured parameters are temperature, relative humidity and height at constant pressure levels. The measured data were provided by the Hellenic National Meteorological Service (HNMS) and come from observations with radio balloons launched twice a day (00 and 12 LT).

The measured parameter values were classified according to observation hour, day of the year and pressure levels and after a preliminary day-by-day statistical process according to the standard deviation of each pressure level, the data were cleared from any unrealistic values. Before calculating the refractivity indices, interpolation procedures were followed to fill in missing values, enrich with data the low altitudes, where the intensity of the tropospheric variations is higher and also to create smoother curves of the initial parameters. The first interpolation procedure was made in a day-by-day manner according to the reference pressure levels and was the most important in the sense that it was applied directly to the initial measured data and provided most of the interpolated values, forming smoothed and enriched curves of temperature, relative humidity and height versus pressure. The next interpolation procedure was also applied each day. Its purpose was to transform the data according to a reference height, common for the whole dataset. This procedure also enriched the low altitude values and also detected and corrected days where abnormal fluctuations of the original data were present. After the analysis of the data according to the above procedures, the refractivity index $N$ was calculated using eqs. (1.1) to (1.4). Finally, an interpolation procedure for $N$ according to time was followed for each altitude level, in order to fill in missing values that could not be interpolated in the previous methods.

For all the interpolation procedures, piecewise-cubic-hermite interpolating polynomials were used, except for high altitudes (higher than $10 \mathrm{~km}$ ), where linear interpolation functions have been applied. This was due to the fact that at high altitudes, when a large number of data were missing, the cubic functions in many cases returned unrealistic results. Spline interpolation functions instead of cubic were also tested, but they resulted in strong oscillations about the measured data, especially in cases where many consecutive values were missing, as is the case for relative humidity. After calculation of refractivity, the scale height $H_{s}$ was determined for each day by applying a least squares nonlinear fitting.

The analysis of the data by means of the previously described procedures and the calculation of refractivity were followed by a statistical evaluation in order to assess their variability.

\section{Results and discussion}

The statistical treatments applied are the second order 31-days moving averages of $N_{0}$ 
and $H_{s}$ versus days and their monthly mean values, together with the corresponding standard deviations. Although calculated $N_{0}$ and $H_{s}$ with respect to time form a time series with periodic variations, moving averages eliminate strong fluctuations by low pass filtering the data. Also, the $\beta_{0}$ parameter was determined for each month for the two observation hours of 00 and 12 LT. This parameter is defined as the percentage of time that the refractive index gradient in the first $100 \mathrm{~m}$ above ground exceeds $100 \mathrm{~N}$ units $/ \mathrm{km}$ and it represents the probability that anomalous propagation may occur.

Figure 1 shows the second order 31-days moving average of $N_{0}$ versus all the days of the period 1991-1999 and for both observation hours (00 and 12 LT). In the horizontal axis the serial number of the day is presented with day 1 corresponding to the 1st January 1991 and Day 3287 to 31 st December 1999. These diagrams constitute a de-noised version of the original calculated refractivity and they show clearly the cycle variations of $N_{0}$ for all years (separated with vertical grid lines). It can be seen that in general, the refractivity increases from January to June, where it appears to reach a first peak. During the summer period, refractivity levels remain high, but a decrement usually occurs during July, reaching a minimum level in August. Then, the refractivity increases till September-October, where it reaches a second peak, higher than the previous one. After October and for the whole winter period, refractivity shows a high rate of attenuation. From the same figure, it can be deduced that only the amplitude of refractivity is changing, being lower at $12 \mathrm{LT}$, while the refractivity variations with respect to time preserve their initial form.

Figure 2 shows the second order 31-days moving average of $H_{s}$ versus the days for the two observation hours of 00 and 12 LT. Comparing this diagram with fig. 1 it can be seen that the scale height follows the form of $N_{0}$, but its variations are lower. The results corresponding to 12 LT are similar to those of 00 LT since the scale height does not show time variations, except for its level which seems higher compared to $00 \mathrm{LT}$.

Further information on seasonal refractivity variations can be extracted from fig. 3 which shows the monthly mean values of $N_{0}$ versus months for the whole analyzed period. In this figure, the increment of the refractivity values during the summer period is clear. This diagram also shows comparative variations of $N_{o}$ for each year. Although all the years do not follow the same level variations, the decrement from June to July is clear, except for 1996, when this phenomenon started on May.

Except for the seasonal variations of refractivity, diurnal variations are also present (fig. 4). This figure shows the standard deviations of the monthly values of $N_{0}$ versus months. This diagram shows that the increase in refractivity

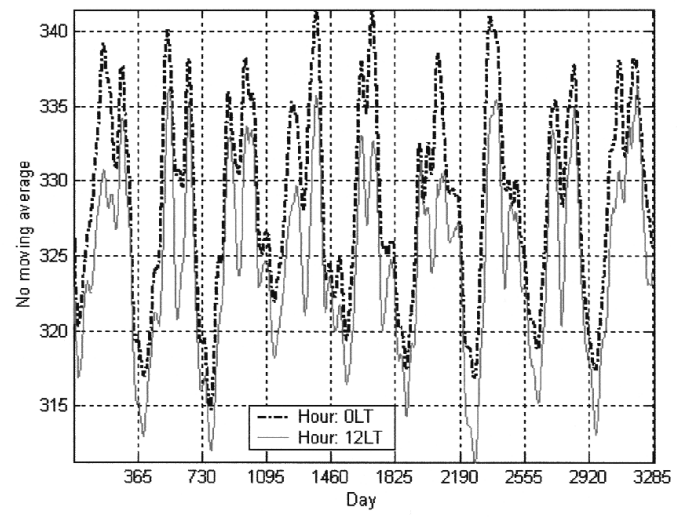

Fig. 1. Second order moving average of $N_{0}$ versus days (observation hour 00 and 12 LT).

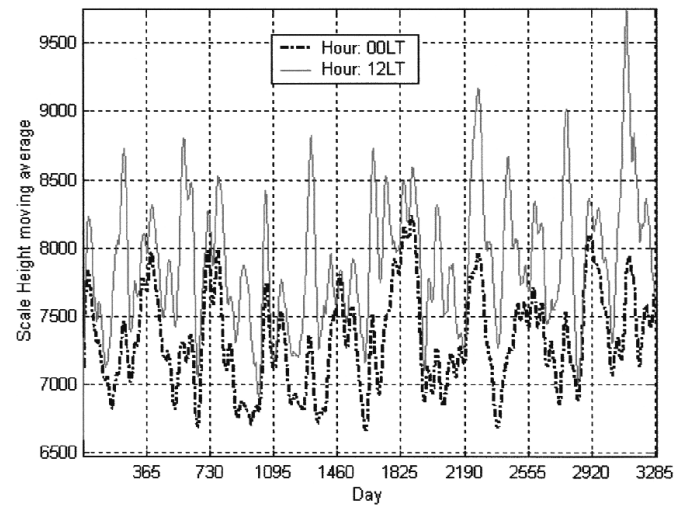

Fig. 2. Second order moving average of $H_{s}$ versus days (observation hour 00 and $12 \mathrm{LT}$ ). 


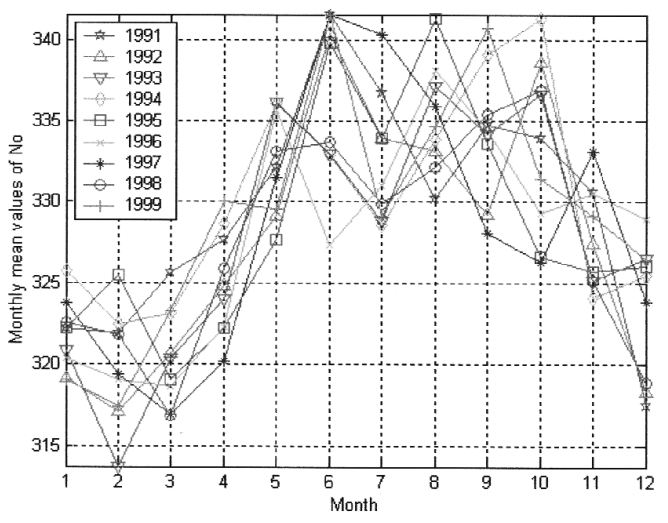

Fig. 3. Monthly mean values of $N_{0}$ versus months (observation hour 00 LT, years 1991-1999).

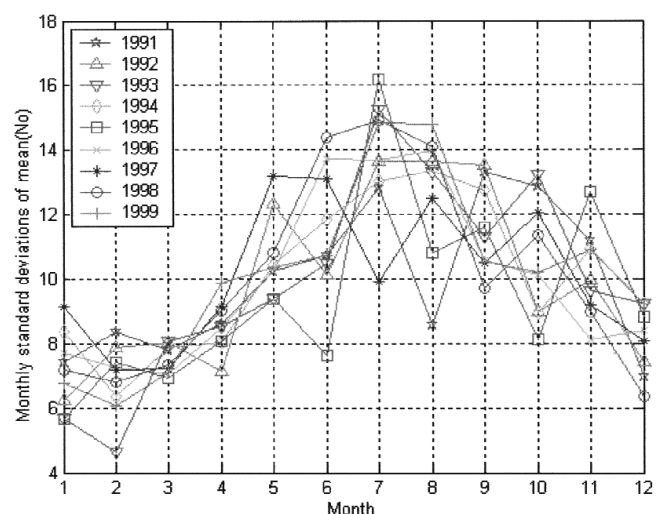

Fig. 4. Standard deviations of the monthly values of $N_{0}$ versus months (observation hour $00 \mathrm{LT}$, years 1991-1999).

levels during the summer is followed by strong deviations within each month. In general, these deviations increase till July where they reach their maximum level and they start to decrease again during the autumn and winter periods.

The identification of the monthly variations of the scale height is not as clear as the variations of $N_{0}$ (fig. 5). This figure shows the monthly mean values of $H_{s}$ versus month for the period 1991-1999. It can be seen that in general $H_{s}$ follows an inverse variation pattern compared to $N_{0}$. It is decreasing during summer and a local

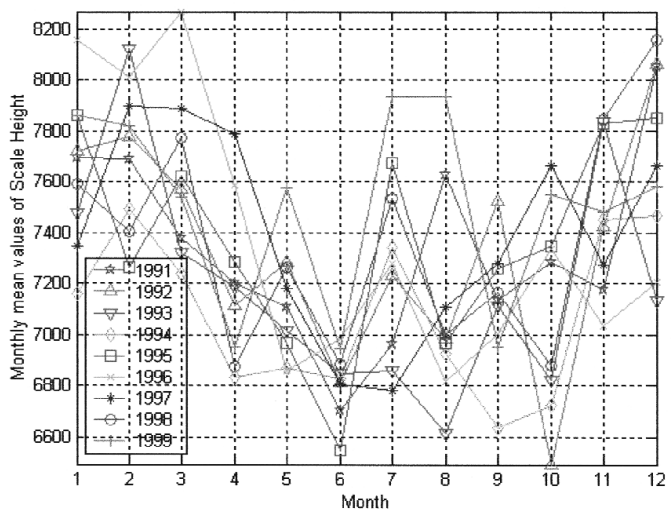

Fig. 5. Monthly mean values of $H_{s}$ versus months (observation hour 00 LT, years 1991-1999).

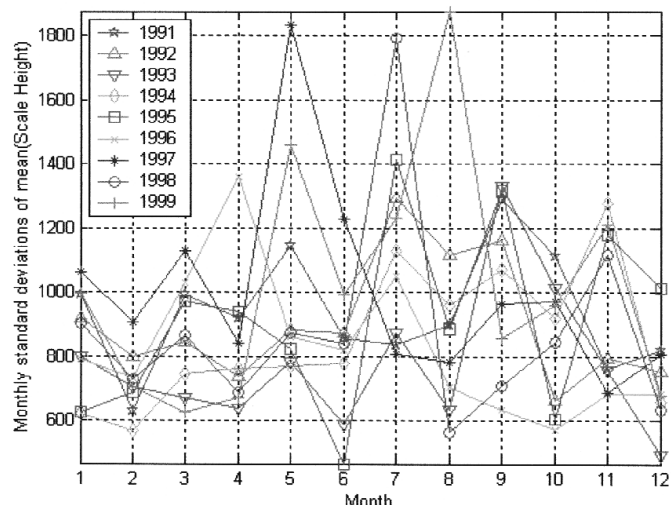

Fig. 6. Standard deviations of the monthly values of $H_{s}$ versus months (observation hour $00 \mathrm{LT}$, years 1991-1999).

peak appears in July but its deviations are very strong and they do not follow the distribution of the standard deviations of $N_{0}$ (figs. 4 and 6).

The results for the $\beta_{0}$ parameter are shown in figs. 7 and 8 , for 00 and 12 LT respectively. By studying fig. 7 it can be seen that the probability of anomalous propagation is very high during the summer months reaching a value of about $90 \%$. During the other periods of the year the $\beta_{0}$ values are much lower but strong variations can be observed in April, September and October where the $\beta_{0}$ values show a fluctuation 


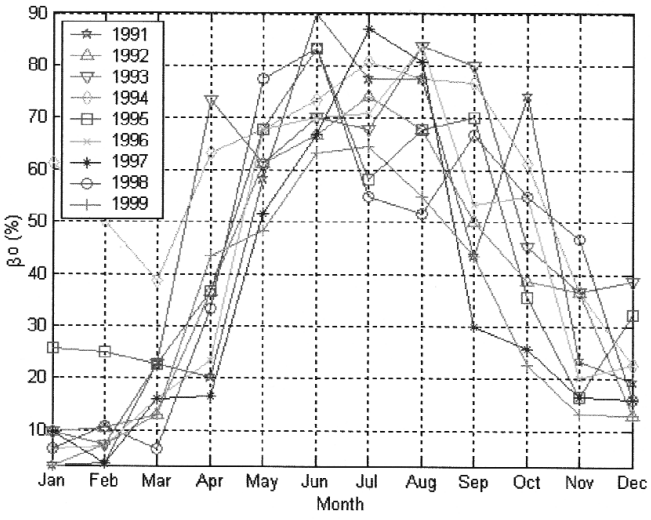

Fig. 7. $\beta_{0}$ parameter versus months (observation hour $=00$ ).

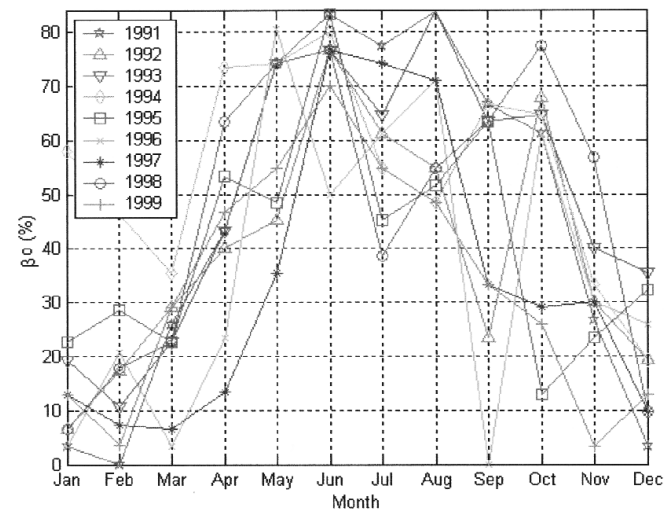

Fig. 8. $\beta_{0}$ parameter versus months (observation hour $=12$ ).

of the order of $50 \%$. For the 12 LT observation hour, it can be seen (fig. 8) that the $\beta_{0}$ values are in general higher during the summer period, but the annual variations are stronger with respect to $00 \mathrm{LT}$ and thus the prediction of anomalous propagation conditions is very difficult.

The relation between $N_{0}$ and $H_{s}$ can be further revealed by comparing their standardized values, $N_{o}^{\prime}$ and $H_{s}^{\prime}$ respectively. The standardization can be made using the equations

$$
N_{0}^{\prime}=\left[N_{0}-\operatorname{mean}\left(N_{0}\right)\right] / \operatorname{std}\left(N_{0}\right)
$$

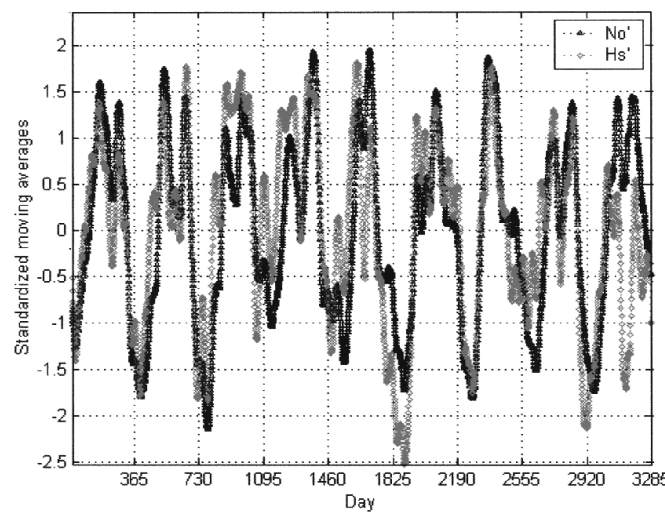

Fig. 9. Comparison of the $N_{0}^{\prime}$ and $H_{s}^{\prime}$ variations (observation hour 00 LT).

$$
H_{s}^{\prime}=\left[H_{s}-\operatorname{mean}\left(H_{s}\right)\right] / \operatorname{std}\left(H_{s}\right)
$$

where the moving averages of $N_{0}$ and $H_{s}$ are used. In the above equations «mean» stands for the global mean value of the moving averages and «std» for their corresponding standard deviation. In fig. 9 the $N_{0}^{\prime}$ and $-H_{s}^{\prime}$ are plotted with respect to days. It can easily be seen that their correlation is very high with respect to time (correlation coefficient $\approx 0.77$ ), whereas $N_{0}^{\prime}$ shows weaker variations.

\section{REFERENCES}

ANDERSON, K.D. (1995): Radar detection of low-altitude targets in a maritime environment, IEEE Trans. Antennas Propag., 43, 609-613.

FLOCK L.W. (1987): Propagation effects on satellite systems at frequencies below $10 \mathrm{GHz}$, NASA Reference Publication Handbook, 1108 (2).

LEAR M.W. (1980): Computing Atmospheric Scale Height for Refraction Corrections, (NASA Mission Planning and Analysis Division, Lyndon B. Johnson Space Center).

Patterson, W.L., C.P. Hattan, G.E. Lindem, R.A. Paulus, H.V. Hitney, K.D. Anderson and A.E. BARrios (1994): Engineer's Refractive Effects Prediction Systems (EREPS) Version 3.0, NRaD Technical Document 2648, May. 
